\begin{tabular}{|c|l|}
\hline Title & Response function measurement of layered type CV D single cry stal diamond radiation detectors for 14 MeV neutrons \\
\hline Author(s) & $\begin{array}{l}\text { Kaneko, J. H.; Teraji, T.; Hirai, Y.; Shiraishi, M.; Kawamura, S.; Y oshizaki, S.; Ito, T.; Ochiai, K.; Nishitani, T.; } \\
\text { Sawamura, T. }\end{array}$ \\
\hline Citation & $\begin{array}{l}\text { Review of Scientific Instruments, 75(10), 3581-3584 } \\
\text { https://doi.org/10.1063/1.1787918 }\end{array}$ \\
\hline Issue Date & 200410 \\
\hline Doc URL & http://hdl.handle.net/2115/5722 \\
\hline Rights & Copyright $\odot 2004$ A merican Institute of Physics \\
\hline Type & article \\
\hline File Information & RSI75-10.pdf \\
\hline
\end{tabular}

Instructions for use 


\title{
Response function measurement of layered type CVD single crystal diamond radiation detectors for $14 \mathrm{MeV}$ neutrons
}

\author{
J. H. Kaneko ${ }^{\text {a) }}$ \\ Graduate School of Engineering, Hokkaido University, N13 W8, Kita-ku, Sapporo 060-8628, Japan \\ T. Teraji \\ Graduate School of Engineering, Osaka University, 2-1 Yamadaoka, Suita, Osaka 565-0871, Japan \\ Y. Hirai, M. Shiraishi, and S. Kawamura \\ Graduate School of Engineering, Hokkaido University, N13 W8, Kita-ku, Sapporo 060-8628, Japan \\ S. Yoshizaki and T. Ito \\ Graduate School of Engineering, Osaka University, 2-1 Yamadaoka, Suita, Osaka 565-0871, Japan \\ K. Ochiai and T. Nishitani \\ Japan Atomic Energy Research Institute, Tokai-mura, Ibaraki 319-1195, Japan \\ T. Sawamura \\ Graduate School of Engineering, Hokkaido University, N13 W8, Kita-ku, Sapporo 060-8628, Japan
}

(Presented on 19 April 2004; published 5 October 2004)

\begin{abstract}
Response function measurement of layered-type chemical vapor deposition single crystal diamond radiation detectors for $14 \mathrm{MeV}$ neutrons was carried out. The detector had a layered structure that was composed of a boron-doped diamond layer of $0.5 \mu \mathrm{m}$ in thickness and a nondoped diamond layer of $20 \mu \mathrm{m}$ on an inexpensive high pressure and high temperature-type Ib diamond substrate. The detector had energy resolution of $2.6 \%$ for $5.5 \mathrm{MeV} \alpha$ particles. This experiment was mainly carried out in order to understand the present status of the detector as a $14 \mathrm{MeV}$ neutron spectrometer and an extent of charge trapping. As result, a peak caused by the ${ }^{12} \mathrm{C}\left(n, \alpha_{0}\right){ }^{9} \mathrm{Be}$ reactions was clearly observed; the best energy resolution of $6 \%$ as for a synthetic diamond radiation detector was achieved. Detection efficiency was $3.2 \times 10^{-7}$ counts/unit neutron fluence. However, taking the energy resolution for $\alpha$ particles, etc., into account, the energy resolution for $14 \mathrm{MeV}$ neutrons was not so high. Further improvement based on better crystal growth is indispensable.
\end{abstract}

(C) 2004 American Institute of Physics. [DOI: 10.1063/1.1787918]

\section{INTRODUCTION}

A diamond radiation detector has several merits in terms of high radiation resistance, ${ }^{1}$ high temperature operation, ${ }^{2}$ high chemical resistance, ${ }^{3}$ etc. Moreover, a diamond radiation detector can be applied to $14 \mathrm{MeV}$ neutron energy spectroscopy using the ${ }^{12} \mathrm{C}\left(n, \alpha_{0}\right){ }^{9} \mathrm{Be}$ reactions. This method corresponds to neutron energy spectroscopy using the ${ }^{28} \mathrm{Si}(n, \alpha){ }^{25} \mathrm{Mg}$ and $\left.{ }^{28} \mathrm{Si}(n, p)\right)^{28} \mathrm{Al}$ reactions in a silicon surface barrier detector. ${ }^{4}$ This capability of a diamond radiation detector was reported by Kovalchuke in 1994 in Ref. 5. The energy resolution of $2 \%$ for $14 \mathrm{MeV}$ neutrons was the best result reported in the previous studies using a natural diamond radiation detector. ${ }^{6}$ This energy resolution satisfied a required capability of a $14 \mathrm{MeV}$ neutron energy spectrometer for ion temperature measurements for DT plasmas. A very compact size of a diamond radiation detector has an advantage in plasma diagnostics that are usually forced strict spatial limitation. Moreover, a diamond radiation detector is at least 100 times tougher than a silicon surface barrier detector in radiation resistance. ${ }^{1}$ As mentioned above, a diamond ra-

\footnotetext{
${ }^{\text {a) }}$ Author to whom correspondence should be addressed; electronic mail:
} kin@qe.eng.hokudai.ac.jp diation detector has ideal properties for a $14 \mathrm{MeV}$ neutron energy spectrometer for plasma ion temperature measurements. However, it was very difficult to routinely produce diamond radiation detectors that had enough performance, because natural diamond crystals for this purpose were very scarce.

On the other hand, a synthetic technique of diamond has made remarkable progress. The authors reported the first trial of $14 \mathrm{MeV}$ neutron energy spectroscopy using a radiation detector made of a synthetic diamond grown by a high pressure and high temperature (HP/HT) method in Ref. 7. Although the detector succeeded to work as an energy spectrometer, there was severe trapping on electrons ${ }^{8}$ and its reduction was indispensable for fruition of a practical detector. Accordingly, characterization of impurities, ${ }^{9}$ applying higher purity diamond crystals ${ }^{10}$ and estimation of charge trapping mechanism into the HP/HT-type IIa diamond crystal $^{11}$ were carried out. At the same time, the application of chemical vapor deposition (CVD) diamond single crystal was carried out successfully. ${ }^{12}$ However, it was revealed that yield rate of CVD single diamond for energy spectrometer was extremely poor. Recently, development of a layered-type CVD single crystal diamond radiation detector was 


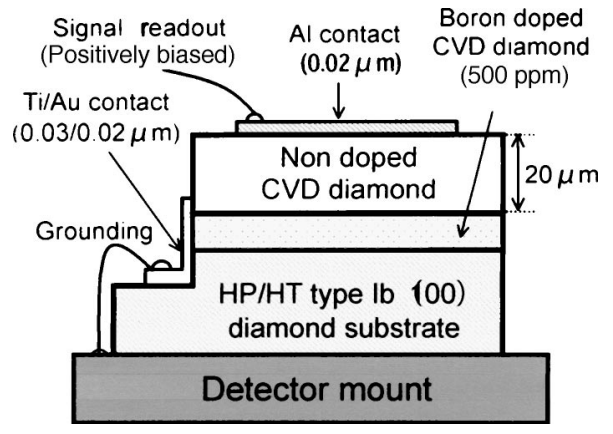

FIG. 1. Schematic drawing of a cross section of a layered-type CVD single crystal diamond radiation detector. The detector was fabricated on an inexpensive HP/HT-type Ib diamond single crystal substrate. The boron-doped CVD diamond layer worked as a contact. The nondoped CVD diamond layer of $20 \mu \mathrm{m}$ in thickness was a sensitive layer.

succeeded, ${ }^{13}$ one main motivation of this development was improvement of yield rate. In this article, response function measurement of a layered-type CVD single crystal diamond radiation detector for $14 \mathrm{MeV}$ neutrons was described. This experiment was carried out in order to obtain a guide to the next improvement, namely fabrication of a thick sensitive layer.

\section{DETECTOR AND EXPERIMENTAL SETUP}

\section{A. Layered-type CVD single crystal diamond radiation detectors}

Figure 1 shows a schematic drawing of a cross section of a layered-type CVD single crystal diamond radiation detector. This detector was developed aiming at improvement of poor yield rate of CVD single crystal diamond radiation detectors and fabrication of a thin sensitive layer enough for $\alpha$ particle energy spectroscopy. ${ }^{13}$ This detector was fabricated on an inexpensive HP/HT-type Ib diamond single crystal substrate, i.e., yellow diamond. The detector had a layered structure composed of a boron-doped single crystal diamond layer of $0.5 \mu \mathrm{m}$ thickness and a nondoped single crystal diamond layer of $20 \mu \mathrm{m}$ on the substrate. These layers were homoepitaxially grown on (100) surface of the substrate by plasma assisted CVD method in order of boron-doped and then nondoped diamond layers. After crystal growth, a part of the nondoped diamond layer was removed by oxygen plasma etching with a metallic mask; at this part, a Ti/Au electrode was fabricated by evaporation, and the borondoped diamond layer worked as a back contact. Moreover, an aluminum Schottky contact was fabricated on nondoped diamond layer by evaporation, too.

Owing to the boron-doped diamond contact, the detectors had strong rectification properties. The detectors had energy resolution of $2.6 \%$ and $2.8 \%$ for $5.5 \mathrm{MeV} \alpha$ particles. $^{13}$ These values were far from higher energy resolution of $0.4 \%$ achieved by radiation detectors made of the HP/HT-type IIa single crystal diamond ${ }^{12}$ and the CVD single crystal diamond. ${ }^{14}$ It had already been known that the quality of the nondoped diamond layers was not very high; it was obvious from their cathode luminescence spectra in which the strong band $A$ luminescence around $420 \mathrm{~nm}$ was observed with the free exciton recombination luminescence at $235 \mathrm{~nm}$, indicat- ing high quality. It was probably caused mainly by poor crystallinity of the boron-doped layer owing to growth instrumentation; the boron-doped layer gave a bad influence on crystallinity of the nondoped layer on it. On the other hand, the detector had a very thin $20 \mu \mathrm{m}$ layer that was very difficult to fabricate by mechanical polishing and a selfstanding diamond substrate, thus there was the possibility of improvement on suppression of charge trapping on electrons that was a big issue with the HP/HT-type IIa diamond. Compared with self-standing CVD single crystal diamonds, the yield rate of the layered-type detector was overwhelmingly high. In this experiment, two layered-type CVD single crystal diamond radiation detectors were used.

\section{B. Experimental setup and measurement electronics}

Response function measurement experiment for $14 \mathrm{MeV}$ neutrons was carried out at the fusion neutronics source (FNS) facility of the Japan Atomic Energy Research Institute. The diamond radiation detector was set at an exit of a $14 \mathrm{MeV}$ neutron collimator. At the position of the detector, peak energy of neutrons was calculated to be $14.2 \mathrm{MeV}$, and it had energy broadening of $1.0 \%$ in full width at half maximum. The detector was connected to a CANBERRA 2003T charge sensitive preamplifier by a cable whose length was $15 \mathrm{~cm}$. A CANBERRA 2021 spectroscopy amplifier and Genie-ESP analysis system were used in this measurement.

\section{EXPERIMENTAL RESULT AND DISCUSSIONS}

Figure 2 shows examples of response functions for $14 \mathrm{MeV}$ neutrons obtained by one of the layered-type CVD single diamond radiation detectors. Bias voltage of $+15 \mathrm{~V}$ $(7.5 \mathrm{kV} / \mathrm{cm})$ was applied to the aluminum contact, and gain and shaping time of the spectroscopy amplifier were 0.6 $\times 1 \mathrm{k}$ and $10 \mu \mathrm{s}$, respectively. Continuous measurement of 120 min was carried out with counting rate of approximately 20 counts/s. Figure 2(a) shows the pulse height spectrum obtained during the first $40 \mathrm{~min}$, and Fig. 2(b) shows the pulse height spectrum obtained during the third $40 \mathrm{~min}$, namely from 80 to $120 \mathrm{~min}$. In Fig. 2(a), basic structure caused by the ${ }^{12} \mathrm{C}\left(n, n^{\prime}\right){ }^{12} \mathrm{C},{ }^{12} \mathrm{C}\left(n, n^{\prime}\right){ }^{12} \mathrm{C}^{*},{ }^{12} \mathrm{C}\left(n, n^{\prime}\right) 3 \alpha$ and ${ }^{12} \mathrm{C}\left(n, \alpha_{0}\right){ }^{9} \mathrm{Be}$ reactions ${ }^{15}$ was clearly observed. In contrast, the structure became dull with increase of measurement time as shown in Fig. 2. It was obvious we had to get the influence of accumulated charge in the detector.

Figure 3 shows a response function measured by another detector. In this measurement, bias voltage of $+25 \mathrm{~V}$ $(12.5 \mathrm{kV} / \mathrm{cm})$ was applied to the aluminum contact. Gain and pulse shaping time constant of the spectroscopy amplifier were $0.6 \times 500$ and $6 \mu \mathrm{s}$, respectively. To obtain this spectrum, six times of a 20 min measurement were summed up. Between each measurement, some bias voltage of opposite polarity was applied to the detector in order to remove space charge. As result, the fine spectrum compared with Fig. 2 was obtained. Moreover, energy resolution of $6 \%$ at the peak caused by the ${ }^{12} \mathrm{C}\left(n, \alpha_{0}\right){ }^{9}$ Be reactions was achieved; it was the best result for synthetic diamond radiation detectors.

Detection efficiency was $2.8 \times 10^{-7}$ counts/unit neutron flux for this detector. Figure 4 shows dependence of detec- 

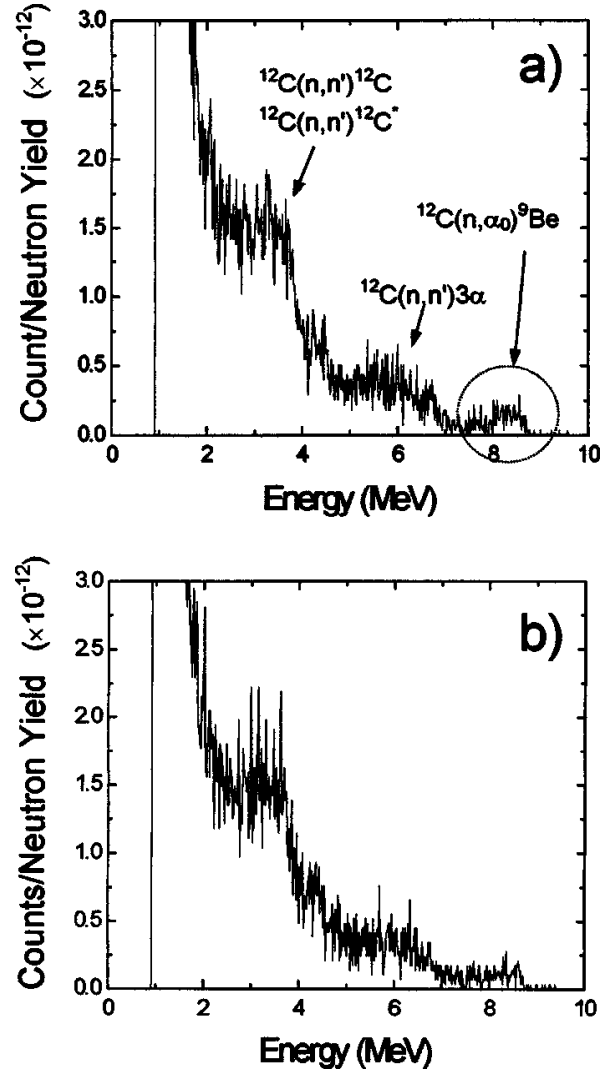

FIG. 2. Change of pulse height distribution spectra obtained by the layeredtype CVD single crystal diamond radiation detector for $14 \mathrm{MeV}$ neutrons due to measurement time. (a) the first $(0-40 \mathrm{~min})$ measurement of series three times measurement in total of $120 \mathrm{~min}$, (b) the third (80-120 min) measurement. Bias voltage of $+15 \mathrm{~V}$ was applied to the aluminum contact. Gain and shaping time of a spectroscopy amplifier was $0.6 \times 1 \mathrm{k}$ and $10 \mu \mathrm{s}$, respectively. It was obvious that spectra were distorted according to the measurement terms.

tion loss on the thickness of a sensitive layer calculated by analytical approximation. Range of $\alpha$ particles created by the ${ }^{12} \mathrm{C}\left(n, \alpha_{0}\right){ }^{9} \mathrm{Be}$ reactions was approximately $15 \mu \mathrm{m}$ in diamond. Thus, for $20 \mu \mathrm{m}$ in detector thickness, approximately $18 \%$ of counts caused by the ${ }^{12} \mathrm{C}\left(n, \alpha_{0}\right){ }^{9} \mathrm{Be}$ reactions was not

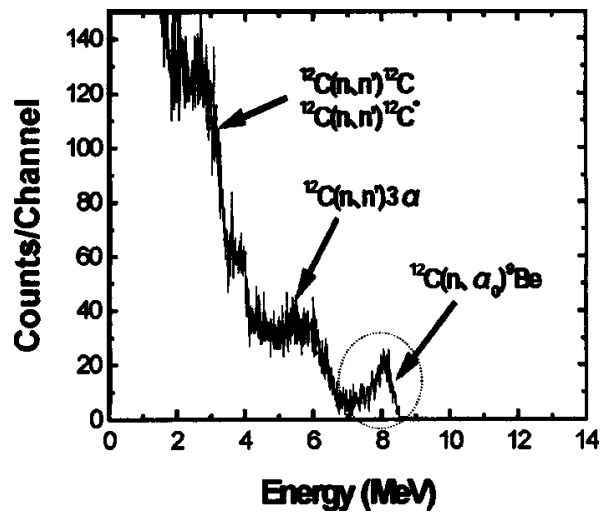

FIG. 3. An example of response function of the layered-type CVD single crystal diamond radiation detector for $14 \mathrm{MeV}$ neutrons. Bias voltage of $+25 \mathrm{~V}(12.5 \mathrm{kV} / \mathrm{cm})$ was applied to the aluminum contact. Gain and shaping time of linear amplifier were $0.6 \times 500$ and $6 \mu$ s, respectively. This spectrum was made by summing up of six times of a 20 min measurement. Between each measurement, bias voltage of opposite polarity was applied to the detector in order to remove space charge in the detector.

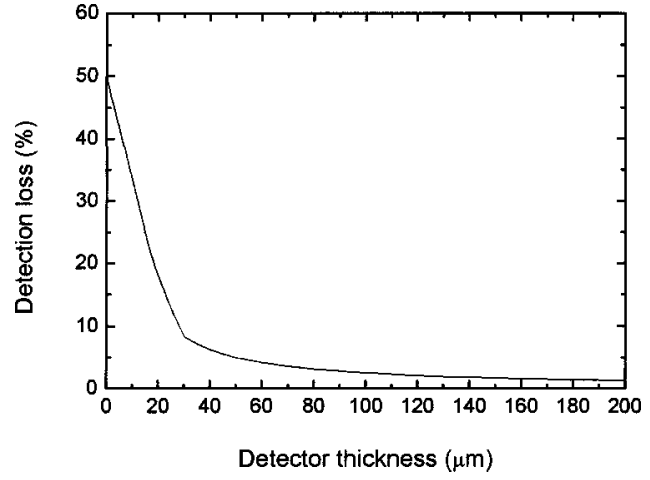

FIG. 4. Dependence of detection efficiency of the ${ }^{12} \mathrm{C}\left(n, \alpha_{0}\right){ }^{9} \mathrm{Be}$ reactions on thickness of sensitive layer of diamond. This figure was calculated using an analytical approximation. The range of $\alpha$ particle produced by the ${ }^{12} \mathrm{C}\left(n, \alpha_{0}\right){ }^{9} \mathrm{Be}$ was approximately $15 \mu \mathrm{m}$. In this calculation, influence of ${ }^{9} \mathrm{Be}$ was ignored, because the range was at most $2 \mu \mathrm{m}$.

able to deposit all the energy to the detector. However, taking the bad energy resolution of approximately 3\% for $\alpha$ particles and the broadening of neutron energy of $1 \%$ into account, calculated detection efficiency of $3.2 \times 10^{-7}$ was almost equal to the experimental result of $2.8 \times 10^{-7}$.

For the detectors used in this study, it was difficult to observe transfer properties of electrons and holes separately, because the thickness of the sensitive layers was almost the same length as a range of a $5.5 \mathrm{MeV} \alpha$ particle, i.e., $14 \mu \mathrm{m}$. If there was no charge collection loss in the detector, approximately $65 \%$ of the pulse height of a signal was composed by motion of electrons for $5.5 \mathrm{MeV} \alpha$ particles. ${ }^{16}$ Moreover, the detectors had strong rectification properties, thus it was impossible to change drift direction of electrons or holes by applying opposite bias polarity. As reported in Ref. 13, in the experimental results obtained using $\alpha$ particles, the detectors had low charge collection efficiency and slow drift velocity of both charges, i.e., $700-2 \times 10^{4} \mathrm{~cm} / \mathrm{s}$. On the other hand, for $14 \mathrm{MeV}$ neutrons, all reactions occurred in the detector homogeneously. Therefore, average contribution to a pulse height of a signal was equal for both electrons and holes. Taking all the information described above into account, there were charge losses not only on electrons but also on holes. Probably, there were trapping centers related to bad crystallinity.

In order to improve detector performance, improvement on the boron-doped diamond layer and optimization for growth condition of nondoped diamond layer are indispensable. For the latter issue, a growth condition achieving higher growth rate of $4 \mu \mathrm{m} / \mathrm{h}$ with very high crystal quality was observed. If this growth condition applied to fabrication of the detector, a $100-\mu \mathrm{m}$-thick detector can be fabricated in $25 \mathrm{~h}$. Therefore, the authors believe that a practical synthetic diamond radiation detector as a $14 \mathrm{MeV}$ neutron energy spectrometer will come about in the near future.

\section{ACKNOWLEDGMENTS}

This research was supported by KAKENHI (15360498). In addition, the experiments at the FNS facility were carried out with a contract from Kyoryoku kenkyu with the Japan Atomic Energy Research Institute. 
${ }^{1}$ S. F. Kozlov et al., IEEE Trans. Nucl. Sci. NS-22, 160 (1975).

${ }^{2}$ F. Nava et al., IEEE Trans. Nucl. Sci. NS-26, 308 (1979).

${ }^{3}$ S. F. Kozlov et al., IEEE Trans. Nucl. Sci. NS-24, 242 (1977).

${ }^{4}$ T. Elevant et al., Rev. Sci. Instrum. 57, 1763 (1987).

${ }^{5}$ V. D. Kovalchuk et al., Nucl. Instrum. Methods Phys. Res. A 351, 590 (1994).

${ }^{6}$ A. V. Krasilnikov et al., Rev. Sci. Instrum. 68, 1720 (1997).

${ }^{7}$ J. Kaneko et al., Rev. Sci. Instrum. 70, 1100 (1999).

${ }^{8}$ J. Kaneko and M. Katagiri, Nucl. Instrum. Methods Phys. Res. A 383, 547 (1996)

${ }^{9}$ J. Kaneko et al., Diamond Relat. Mater. 9, 2019 (2000).
${ }^{10}$ T. Tanaka et al., Rev. Sci. Instrum. 72, 1406 (2001).

${ }^{11}$ J. H. Kaneko et al., New Diamond Front. Carbon Technol., 14, p. 299 (2004).

${ }^{12}$ J. H. Kaneko et al., Nucl. Instrum. Methods Phys. Res. A 505, 187 (2003).

${ }^{13}$ J. H. Kaneko et al., J. Jap. Atom. Energy Eng. (submitted).

${ }^{14}$ J. Kaneko et al., Nucl. Instrum. Methods Phys. Res. A 422, 211 (1999).

${ }^{15}$ M. Pillon et al., Nucl. Instrum. Methods Phys. Res. B 101, 473 (1995).

${ }^{16}$ G. F. Knoll, Radiation Detection and Measurement, 2nd ed. (Wiley, New York, 1988), p. 421. 